\title{
Magnetic anomalies of steel drums: a review of the literature and research results of the INGV
}

\author{
Marco Marchetti, Vincenzo Sapia, Alessandro Settimi
}

Istituto Nazionale di Geofisica e Vulcanologia, Sezione di Geomagnetismo, Aeronomia e Geofisica Ambientale, Rome, Italy

\author{
Article history \\ Received September 6, 2012; accepted December 11, 2012. \\ Subject classification: \\ Toxic waste, Steel-drum detection, Magnetic anomalies.
}

\begin{abstract}
The detection and evaluation of the status of disposal sites that contain hazardous waste materials is becoming an increasingly important element in environmental investigations. Close cooperation between the Istituto Nazionale di Geofisica e Vulcanologia (INGV; National Institute of Volcanology and Geophysics) in Rome and the Italian environmental police has resulted in numerous underground investigations of different buried materials. Among the geophysical investigation tools, magnetometry is the most effective, rapid and precise of all of the geophysical methods for localizing buried steel drums. Analysis of magnetic map anomalies can provide a variety of information about buried materials, including extension, distribution and depth, with processing of the acquired magnetic data. This information is also very useful in case of excavations that are aimed at the recovery of hazardous waste. This study determines the most relevant analyses reported in the literature, with modeling of magnetometric methods for environmental applications both theoretically and experimentally. Some studies and research results achieved by the INGV in relation to magnetic anomalies produced by buried steel drums are also reported, as found in field operations and as achieved from test sites.
\end{abstract}

\section{Introduction}

Underground disposal of steel drums that contain hazardous waste materials and illegal dumping of waste or toxic fluids can harm the environment. Dangerous buried substances and leakage of fluids contained in drums can heavily contaminate agricultural land and pollute groundwater resources; the food chain can be altered, posing a serious risk to human health and the ecosystem.

This issue is quite relevant in Italy. Each year the Ecomafia report (edited by Legambiente) details the trafficking and illegal disposal of waste and the investigations of the environmental police. Since 1994, four Parliamentary Commissions of Inquiry have been set up to expose the covert waste disposal and related illegal activities.

Localization and evaluation of disposal sites are the primary aims in environmental investigations. Generally, these include fly-tipping sites, abandoned quarries for land fill, or simple agricultural land. Often, the recovery of the grass hides excavation activities and the investigations require more than simple observations of the ground. After locating illegal dumping sites, to assess the possibility of pollution at the site and to monitor its development through time, the extent of land affected by disposal, the bulk and nature of the buried materials need to be are established.

Excavations can be conducted at anomalous sites identified by geophysical measurements, which provides information to guide the excavations and to reduce the risk of damaging the recovered drums of toxic waste.

\section{Magnetometry}

The magnetometric technique is the most frequently used geophysical method for environmental investigations [Bevan 1983, Tyagi et al. 1983, Barrows and Rocchio 1990, Roberts et al. 1990, Schlinger 1990, Gilkenson et al. 1992, Foley 1994, Cochran and Dalton 1995, Gibson et al. 1996, Ravat 1996, Marchetti et al. 1998, Eskola et al. 1999, Furness 1999, Godio 2000, Furness 2001, Furness 2002, Marchetti et al. 2002, Salem et al. 2002, Furness 2007, Sheinker et al. 2009]. Among the potential techniques for geophysical exploration of the subsoil, magnetometry is generally acknowledged to be among the most effective, rapid and precise for the identification of buried ferromagnetic masses [Marchetti 1997, Marchetti and Meloni 1997, Marchetti 2000]. Magnetometric surveys detect the surface effects and local disturbances to the Earth's magnetic field that can be caused by buried ferromagnetic objects. These effects are referred to as magnetic anomalies, and they are a consequence of the interaction of the Earth's magnetic field with both the induced and permanent magnetization of the magnetic targets. Natural bodies (like magnetic ore deposits) and man-made iron and steel objects (including pipelines, vehicles, railways, mines, and as in the present case, buried storage drums) can generate deformations in the local geomagnetic field. The possibility of detecting magnetic objects with a magnetometer depends 
on the effective magnetic mass, the intensity of magnetization, and the distance from the magnetometer. For materials of the same susceptibility, the intensity of an anomaly varies inversely to the square (for a monopole) or the cube (for a dipole) of the distance [Breiner 1973].

An induced magnetic anomaly varies according to the shape, dimensions and amplitude, depth, orientation, geometry and magnetic susceptibility of the body (here as the configuration of steel drums). It is also influenced by the intensity and inclination of the Earth's magnetic field in the survey area. Anomaly values can be negative when the measured field is lower than expected, or vice versa, positive. In the northern hemisphere, buried iron metallic objects normally generate dipolar anomalies, with a positive response south and a negative response north of the source [Marchetti et al. 1996, Marchetti and Meloni 1997, Marchetti et al. 1998].

Geologists have traditionally used magnetic surveys to identify changes in rock types, to indicate, for example, the presence of ore bodies, fault lines or igneous intrusions. The technique is also commonly applied to estimate the magnetic base depth beneath sedimentary rock. In these applications, the distribution of ferromagnetic minerals (mostly magnetite) within the terrain represents the principal geological variable. The theory behind these methods and the survey procedures have been detailed in a number of reference texts [Grant and West 1965, S.E.G. 1967, Nettleton 1976, Telford et al. 1976, Parasnis 1986, Robinson and Coruh 1988, Vogelsang 1994, Huang and Keiswetter 1997, Chianese et al. 2006a, Chianese et al. 2006b]. These applications can normally detect shallow iron or steel materials, and this influences the way the surveys are conducted and interpreted [Tyagi et al. 1983, Frischknecht and Raab 1985, Gilkeson et al. 1986, Jachens et al. 1986, Gilkeson et al. 1992, Pierce and De Reamer 1993, Godio et al. 1999].

Ravat et al. [2007] critically tested the actual performance of several spectral magnetic depth-determination methods in terms of their capacity to determine the depth to the magnetic base for random and layered model magnetic sources of variable thicknesses. They showed that in some cases, despite various precautions, the results can be erratic, and so a critical evaluation of the results is advisable, with heat-flow modeling, and when possible taking seismic data into account regarding crustal and lithospheric thicknesses and seismic velocities.

\subsection{Permanent and induced magnetization}

Iron objects generally have both permanent and induced magnetization, which combine into a net magnetization that shows a single Earth's magnetic field anomaly, as measured by a magnetometer. In the following rules, it is assumed for simplicity that an anomaly is produced by induced moment only. However, the harder the steel material is, the greater its permanent magnetization, which can sometimes amounting to 10-fold (or even more) the induced magnetization. While the orientation of the permanent moment of buried materials usually cannot be predicted, it can be assumed that the greater the permanent magnetization, the greater the anomaly. The susceptibility, $k$, in the formulae below is really an effective $k$, and it is understood to include any such increased magnetization. A single large item, like a pipe or an engine, for instance, might exhibit a single anomaly largely due to the permanent moment. A very concise review of surveying with portable magnetometers was provided by Breiner [1973].

Conversely, the magnetic effects of drum accumulations are assumed to be generated only by induced magnetization. Ignoring the permanent magnetization in this way does not suggest that it is absent in thin-walled steel drums. Eskola et al. [1999] have reported ample evidence of its presence. However, the principal component of permanent magnetization is acquired before fabrication, and its effect in the completed drum is likely to be diminished by mutual compensation of the effects of the three basic components of a drum, which is composed of the two covers and a cylinder wall. The case for ignoring the effects of permanent magnetization becomes more obvious with multiple drums. Accumulations of steel drums probably constitute one of the few source bodies in magnetic exploration in which it is justifiable to ignore permanent magnetization.

It should be noted that the present discussion refers to a single body, while general theory extends to multiple bodies in an obvious way. The magnetic body is subjected to a primary magnetic field $\vec{H}_{p}$, which is considered to be generated by sources external to and remote from the body.

In line with Stratton [1941], it is assumed that there are no conduction currents in the zone of interest, and so the magnetic field $\vec{H}$ is irrotational and can be identified with the gradient of a scalar magnetic potential $\phi$; i.e.:

$$
\vec{H}=-\nabla \phi
$$

The magnetic induction field $\vec{B}$ arises from both the $\vec{H}$ field and from the magnetization (induced and permanent). According to Stratton [1941], this can expressed by:

$$
\vec{B}=\mu_{0} \vec{H}+\mu_{0}(\vec{M}+\vec{R}),
$$

where $\mu_{0}$ is the permeability of free space, and $\vec{M}$ and $\vec{R}$ are the induced and permanent magnetization, respectively.

The fields are so small in the geophysical environment that the induced magnetization is linearly related to the inducing field $\vec{H}$; i.e.:

$$
\vec{M}=k \vec{H},
$$

where $k$ is the susceptibility. Application of the above Equations (1), (2) and (3) leads to: 


$$
\vec{B}=-\mu \nabla \phi+\mu_{0} \vec{R}
$$

where $\mu$ is the permeability, which is defined by:

$$
\mu=\mu_{0}(1+k)
$$

Finally, a magnetic body of uniform susceptibility $k$ located in free space and influenced by a primary magnetic field $\overrightarrow{H_{p}^{\prime}}$ is considered here. Eskola [1992] noted that the influence of uniform permanent magnetization $\vec{R}$ in a body of constant susceptibility $k$ enclosed in free space is equivalent to the effect of a primary field, given by $\vec{R} / k$, which acts in the absence of permanence. Consequently, the linearity of the primary magnetic field means that the effect of a permanent magnetic field in the present model can be established by applying a modified primary magnetic field, given by:

$$
\vec{H}_{p}^{\prime}=\vec{H}_{p}+\vec{R} / k
$$

in all of the equations.

\subsection{The microscopic origin of magnetism}

The microscopic origin of magnetism was discussed by Parkinson [1983], Rikitake and Honkura [1985], Lanza and Meloni [2006], and Gubbins and Herrero-Bervera [2007]. There are two sources of magnetic moments in electronic motions, due to orbital and unpaired spins. These moments respond to external magnetic fields, which results in induced magnetization. There are three causes of atomic magnetic moments: first, a current circuit formed by orbiting electrons; secondly, an inherent magnetic moment of electrons associated with and parallel to the atomic spin; and thirdly, the inherent magnetic moment of the nucleus (this last considered to be minor, and can be ignored for the present purposes) [Goldestein and Ward 1966]. Orbital moments are an important means for coupling spin moments to a crystal lattice, and so most properties of ferromagnetic substances can be explained by the inherent magnetic moment of the electrons (the spin moment). Ferromagnetic phenomena are largely controlled by a variety anisotropies that are associated with crystal structure or body shape (magneto-crystalline anisotropy) [Jackson 1991]. This means that the electron spin direction is influenced by crystal structure, and energy is dependent on the direction of spontaneous magnetization relative to the crystal lattice axes.

The magnetic moment of atoms in a ferromagnetic material causes them to behave similarly to tiny permanent magnets. They adhere together and align more or less uniformly in small regions known as magnetic domains, or Weiss domains. When exposed to a magnetic field, the domain boundaries shift, and the domains aligned with the magnetic field extend and dominate the structure. When the magnetizing field disappears, the domains might not return to the unmag- netized state. In this way, some materials are magnetized, creating permanent magnets. These substances are called ferromagnetic materials sensu latu, and they can be subdivided into pure ferromagnetic, ferromagnetic and anti-ferromagnetic, according to their spin moment direction and relative intensity. Spin moments can be all mutually parallel and concordant, or all mutually anti-parallel, or not equal and anti-parallel. Each ferromagnetic substance has a specific temperature, called the Curie temperature, or Curie point, above which it loses its ferromagnetic properties. When a magnetized ferromagnetic material is heated to the Curie point temperature, the magnetic domains lose their organization and the magnetic properties are dispersed. If the material is then cooled, the domain alignment structure is spontaneously restored.

An interaction energy, $E_{H}$, acts between the magnetization of individual ferromagnetic particles, $\vec{M}_{i}$, and an applied magnetic field, $\vec{H}$. The interaction energy describes how the magnetization of a ferromagnetic grain is influenced by a magnetic field applied externally. The interaction energy, $E_{H}$, is given by [Lanza and Meloni 2006]:

$$
E_{H}=-\frac{\vec{M}_{i} \cdot \vec{H}}{2} .
$$

This energy density (energy per unit volume) applies to both single-domain (SD) and multi-domain (MD) grains.

Single-domain grains show uniform magnetization, with $M_{i}=M_{s}$. Consequently, the application of a magnetic field cannot change the magnetization intensity, but can rotate $\vec{M}_{s}$ in the direction of the applied field. However, there is resistance to the rotation of $\vec{M}_{s}$. The resistance comprises different components, known as anisotropies, which result in energetically preferred directions for $\vec{M}_{s}$ within individual SD grains.

A highly elongated ferromagnetic grain has much lower magnetostatic energy when magnetized along its length, rather than perpendicular to its length. This is because the proportion of surface covered by the magnetic charges is relatively small when $\vec{M}_{s}$ runs along the major dimension of the grain. Therefore, the internal demagnetizing factor along the principal axis, $N_{D D}$, is much lower than the internal demagnetizing factor perpendicular to the principal axis, $N_{D p}$.

The difference in magnetostatic energy between magnetization along the principal axis and magnetization perpendicular to the principal axis can be defined as [Lanza and Meloni 2006]:

$$
\Delta E_{m}=\frac{\left(H_{D p}-N_{D l}\right) M_{s}^{2}}{2}=\frac{\Delta N_{D} M_{s}^{2}}{2},
$$

where $\Delta N_{D}$ is the difference in demagnetizing factors between the shortest and longest axes. This magnetostatic energy difference represents an energy barrier to the rotation of $\vec{M}_{s}$ in the perpendicular direction. In the absence of other influences, the grain will have $\vec{M}_{s}$ along the principal axis. 


\section{The magnetic fields of steel drums}

Over recent decades, there has been a trend towards increased use of geophysical surveys for environmental monitoring and remediation purposes. A common technique applies magnetics to locate man-made ferromagnetic objects, including steel drums used as containers for various hazardous waste materials. The location of disposal sites and their assessment has become an increasingly important aspect of environmental investigations.

Typically, a waste disposal site will have numerous containers buried at shallow depth, and so localization is relatively simple. The main point of interest is determining the horizontal position and lateral extent of the drums, which is generally obvious on the basis of the magnitude of the magnetic response measured directly above the buried containers. However, the secondary objective of using the observed magnetic anomalies to establish the number of containers is more problematic.

The magnetic survey method is now well established in environmental and engineering investigations for detecting ferromagnetic artefacts. Disposal sites are commonly targeted in these investigations, to identify buried steel drums that hold hazardous waste materials. Geophysics is often required to locate these containers and to estimate their quantity. Magnetic surveys for this purpose must be specifically designed and the resulting data carefully interpreted. For these surveys to succeed, it is necessary to model the magnetic fields generated by thin-walled ferromagnetic containers (typically steel drums). Unfortunately, this has not proven easy to achieve, with two obvious reasons for the lack of progress.

First, the geophysical literature provides little information about the basic magnetic parameters of steel drums (i.e., their susceptibility and permanent magnetic fields). Initial attempts to measure the susceptibility of small steel drum samples through the application of conventional laboratory techniques were made by Emerson et al. [1992] and Ravat [1996]; however, these were not successful due to the effects of demagnetization. Later, Eskola et al. [1999] described a different technique that used a flux gate magnetometer, which is suitable for measuring both susceptibility and permanent magnetization of thin ferromagnetic materials. Unfortunately, the literature includes the properties of only $13 \mathrm{sam}$ ples that have been measured using this technique.

It is of note that some useful information on the magnetic characteristics of steel drums has also been obtained by field measurements. The effects of induced and permanent magnetization have been discriminated by observing the magnetic responses of drums in several orientations [Emerson et al. 1992, Ravat 1996]. The two components of magnetization can be established in terms of equivalent dipoles by inversion of the field data. For a given volume of ferromagnetic material, the ambient magnetic field $\vec{H}$ allows calculation of the magnetization and susceptibility. These data are useful for equivalent source modeling of the magnetic fields produced by steel drums, but they do not provide the true or intrinsic parameters of the ferromagnetic materials used in their construction.

The second and perhaps most significant reason for a lack of progress towards a practical magnetic model for steel drums is the difficulty of modeling the magnetic fields of thin sheets with very high magnetic susceptibility. Eskola et al. [1993] demonstrated the inappropriateness of standard modeling algorithms for this purpose. As a result of this modeling problem, a range of approximations can be applied for the magnetic responses of steel drums. The field measurements of Emerson et al. [1992] and Ravat [1996] have been explained in terms of equivalent dipoles or bodies of simple shape, and magnetized along the direction of the Earth's field. These techniques have proven useful for predicting magnetic profiles located relatively remote from a drum, but they are ineffective for predicting amplitude.

The application of exact numerical (surface integral equation) models has also been unsuccessful. The problem is the numerical instability of these models when applied to very thin and intensely magnetized sheets [Eskola et al. 1993, Traynin and Hansen 1993]. However, one general surface integral equation technique has proven to be relatively robust in this respect. The capacity of a general surface integral equation formulation to deal with thin-walled ferromagnetic bodies was demonstrated by Furness [1999, 2001, 2002, 2007]. The technique was originally described in an electrostatic context by Phillips [1934]. Furness [2002] computed the sum of contributions from small segments of a cylinder. Before that, Singh and Sabina [1978] proposed a closed form formula of the magnetic field produced by a right vertical cylinder. Their study has been frequently used for geophysical prospecting in the past.

It is worth noting that there are two other options in the geophysical literature for the precise numerical modeling of drum responses. Both the specialized integral equation technique described by Nabigian et al. [1984], and the integrodifferential equation approach of Eskola et al. [1989] and Eskola [1992] were designed specifically to address the type of thin, highly susceptible bodies of interest here.

\subsection{Approximate modeling of drum responses}

In response to the complexities of more precise modeling procedures, it is common to apply a number of equivalent source modeling techniques to steel drums [e.g., Emerson et al. 1992, Ravat 1996]. In these techniques, the source body is represented by simplified shapes; e.g., cylindrical prisms and spheres magnetized in the direction of the Earth's field. The practical results satisfactorily predict the shape of the measured anomaly profiles. However, the measurement of magnitudes is poor, and consequently an equivalent susceptibility is generally estimated by matching 
measured and modeled responses.

Furness [2002] reviewed the application of these techniques in terms of their computational efficiency, compared to the exact integral equation method. Figure 1 shows a north-south profile above a vertical standard steel drum that was computed using a variety of techniques. Curve (a) in Figure 1 shows the exact anomaly profile as computed by Phillip's integral equation technique using 1440 planar-surface elements. This shows a typical dipolar response, with total amplitude in the vicinity of $160 \mathrm{nT}$.

The first set of approximate profiles (Figure 1, curves (b), (c)) are the results of approaches that recognize the true drum geometry (i.e., a hollow cylindrical prism), but approximate the magnetization with various simplifying assumptions. These profiles were computed by making the general expression more specific to the magnetic field generated by a drums; i.e.:

$$
\vec{H}(\vec{r})=\vec{H}_{p}(\vec{r})-\frac{1}{4 \pi} \nabla \iiint_{V} \vec{M}\left(\vec{r}^{\prime}\right) \cdot \nabla^{\prime} \frac{1}{\left|\vec{r}-\vec{r}^{\prime}\right|} d v^{\prime},
$$

for the case of an approximating $N$-sided plane polyhedral shell with wall thickness $t$. This results in [Furness 2002]:

$$
\begin{aligned}
\vec{H}(\vec{r}) & =\vec{H}_{p}(\vec{r})-\frac{t}{4 \pi} \sum_{i=1}^{N} \nabla \\
& {\left[M_{n i} \iint_{\Delta S_{i}} \hat{n} \cdot \nabla^{\prime} \frac{1}{\left|\vec{r}-\vec{r}^{\prime}\right|} d s^{\prime}+M_{l i} \sum_{j=1}^{N_{i}} \int_{\Delta C_{i j}} \frac{\hat{l} \cdot \hat{m}_{i j}}{\left|\vec{r}-\vec{r}^{\prime}\right|} d c^{\prime}\right], }
\end{aligned}
$$

where $\Delta C_{i j}$ is the $j$ th edge of the $i$ th polygonal face with median surface $\Delta S_{i}, M_{n i}$, and $M_{l i}$ are the normal and longitudinal components of the magnetization in the wall of the $i$ th face [Eskola et al. 1993]. Equation (10) is easily evaluated by identifying the first integral term as the field due to a uniform double layer on the plane polygonal surface $\Delta S_{I}$, and the second as the field due to a uniform line source on the straight edge $\Delta C_{i j}$.

Curve (b) in Figure 1 is the result of the assumption that the secondary magnetic field is negligible compared to the primary component, when magnetization is given by:

$$
\vec{M}=k \vec{H}_{p} .
$$

The form of this curve reasonably approximates that of the true response, but the true amplitude is seen to be grossly overestimated. This is understandable considering that the above assumption neglects demagnetization completely.

Curve (c) in Figure 1 is the result of an attempt to account for demagnetization. It is assumed that the local demagnetizing field associated with a planar polygonal sheet of the hollow polyhedron is equivalent to that of an infinite sheet with zero depolarization factor in the longitudinal direction, and a unit depolarization factor in the normal direction. Accordingly, the components of magnetization in the directions normal and parallel to the face are assumed to be given by [Furness 2002]:

$$
\vec{M}_{n}=\frac{k}{1+k} \vec{H}_{p n}
$$

and

$$
\vec{M}_{l}=k \vec{H}_{p l},
$$

where $\vec{H}_{p n}$ and $\vec{H}_{p l}$ are the corresponding components of the primary magnetic field. Again, the resulting profile shape (Figure 1, curve (c)) is a good approximation of the true profile, but it still has excessively high amplitude.

The next set of profiles (Figure 1, curves (d), (e)) was generated by representing the magnetic effect of the drum as an equivalent dipole. Accordingly, the anomalous magnetic field is simply given by:

$$
\vec{H}(\vec{r})=-\nabla\left(\frac{1}{4 \pi} \vec{m} \cdot \nabla^{\prime} \frac{1}{\left|\vec{r}-\vec{r}^{\prime}\right|}\right),
$$

where $\vec{r}$ ' is the position vector of the dipole with moment located at the centroid of the drum.

Curve (d) in Figure 1 is the result of approximating the dipole moment to that of a thin spherical shell with equal surface area and wall thickness; i.e., a shell with a metal volume equal to the drum. This provides a greatly improved fit for the theoretical profile compared with previous approximations, although with somewhat reduced amplitude.

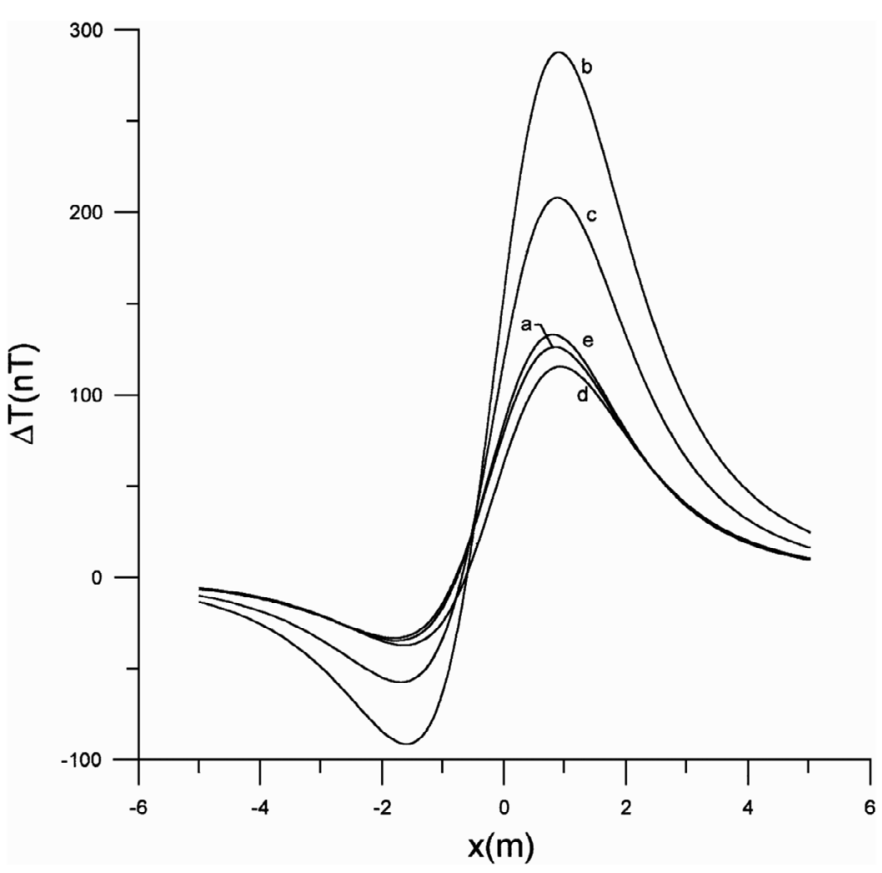

Figure 1. Total magnetic field profile over a vertical standard drum buried $2 \mathrm{~m}$ below the sensor height, computed by the exact integral equation technique (a), ignoring demagnetization effects (b), use of local depolarizing factors (c), use of the dipole moment due to an equivalent spherical shell (d), and use of a dipole moment computed from the longitudinal and transverse apparent susceptibilities of a standard drum (e). Reproduced from Furness [2002]. 
(a)

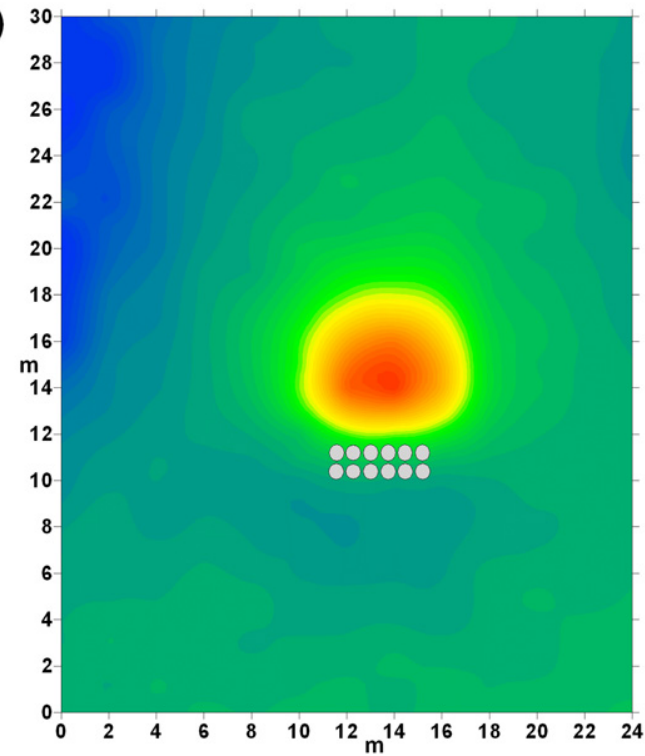

(b)

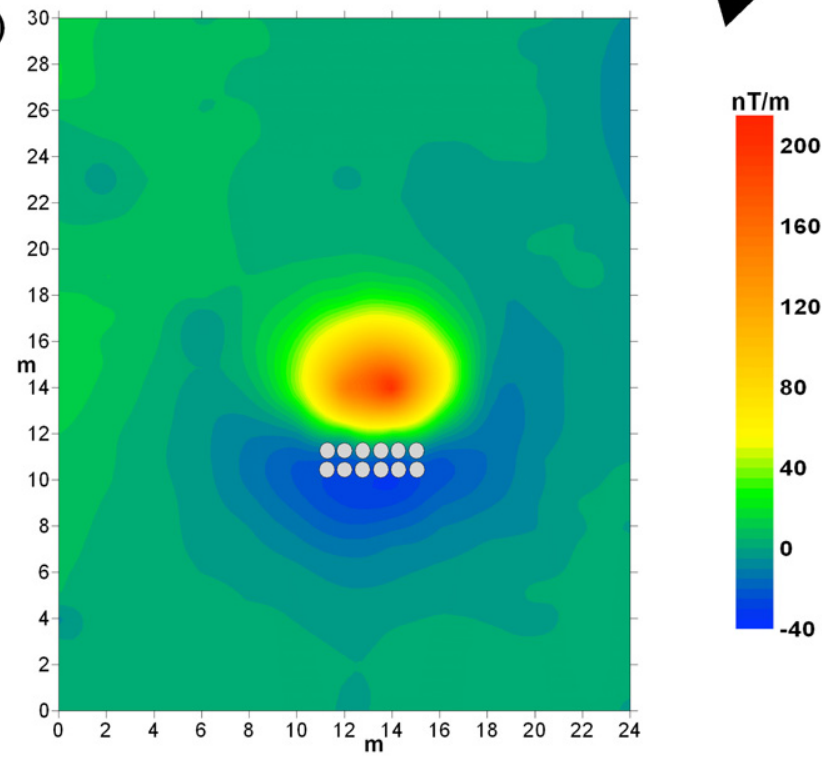

Figure 2. The test site with 12 buried drums. The magnetic inclination was around $58^{\circ}$. (a) Anomaly map of the total intensity field. (b) Map of the vertical gradient.

Finally, curve (e) in Figure 1 is the result of applying the apparent longitudinal and transverse susceptibilities computed for the standard drum in Furness [2002]. The longitudinal and transverse components of the equivalent dipole moment are therefore given by:

$$
\vec{m}_{l}=k_{\alpha l} \vec{H}_{p l} V
$$

and

$$
\vec{m}_{t}=k_{\alpha t} \vec{H}_{p t} V
$$

where $V$ is the volume of metal, $k_{\alpha l}$ and $k_{\alpha t}$ are the apparent longitudinal and transverse susceptibilities, the values of which are taken from Figure 1.

This last curve (Figure 1, curve (e)) provides the best fit to the true response profile of all of these approximations in terms of both amplitude and shape. However, the amplitude is seen to be somewhat underestimated; this is the result of higher multipoles in the response behavior, which are not accommodated by the apparent susceptibilities.

\section{The studies performed by the INGV}

In recent years, the Istituto Nazionale di Geofisica e Vulcanologia (INGV; National Institute of Volcanology and Geophysics) prepared two test sites to examine anomalies produced by underground steel drums. The first test was carried out by burying 12 new 55 gallon steel drums in an area characterized by fluvial-glacial deposits that consist of conglomerates in a silt-sandy matrix. Measurements performed at this test site using a proton precession magnetometer in gradiometric configuration allowed the determination of the magnetic signatures of these 12 unrusted buried steel drums. Furthermore, suitable modeling revealed the magnetic response of the metallic bodies at different depths [Marchetti et al. 1998] (Figure 2).

A second test site was set-up in clayey-sandy deposits. Twelve empty steel drums, oriented vertically, were buried with their top surface at a depth of $4 \mathrm{~m}$ to $5 \mathrm{~m}$ below ground level. The magnetic data were collected using an optical pumped caesium magnetometer in gradiometer configuration. The typically measured dipolar magnetic anomaly was characterized by a well-defined maximum and a less intense minimum; this anomaly reached a total intensity of about $290 \mathrm{nT}$, and its main axis was oriented North-South (N-S). The vertical gradient more precisely characterized the steel drum anomaly. Indeed, it can detect shallow buried targets better than the total intensity magnetic field [Marchetti and Settimi 2011]. An assemblage of steel drums can be viewed as the combination of single individual permanent magnetizations that partly compensate each other, leaving almost only the induced component. The combination of residual magnetization vectors due to each frame would lead to an array of negligible intensities compared to the predominant induced magnetization of the whole body, so the anomalies are aligned in an almost S-N direction (northern hemisphere). Theoretically, the permanent contribution can be drastically reduced for a large number of drums.

The magnetic field produced by a single drum was also investigated; magnetometric measurements on a single metal drum with reconstruction of the field anomaly produced in the surrounding space were performed by Marchetti et al. [1996]. In particular, magnetic measurements were performed along N-S profiles at various distances from a single drum lying on the ground. For each profile, the drum was rotated $180^{\circ}$ around its axis in the four possible dispositions. This operation was repeated aligning the major axis of the drum along both the N-S and E-W directions. The anomaly profiles carried out at a distance of $2 \mathrm{~m}$ from the drum were substantially similar in shape in the dispositions 
obtained by rotating the drum around its minor axis. Instead, the anomaly profiles showed a rather different form in the dispositions obtained by rotating the drum around its major axis. In practice, the anomaly profile changes completely with the rotation of the drum around the principal axis (oriented N-S), whereas this is not the case for the rotation of the drum around the minor axis (except for a small change in anomaly intensity).

Furthermore, two symmetrical profiles of a drum were taken, as the profiles parallel to longest side of a drum and at a distance of $2 \mathrm{~m}$ from the drum. The drum was rotated as in the various dispositions described above. Small magnetic anomaly maps of the area around the drum were obtained from these data, with one for each rotation. Essentially, the same phenomena were observed appearing with magnetic profiles, but, in this case, the effects were more obvious and were related to the space surrounding the drum. A single drum alters the force lines of the Earth's magnetic field, which produces anomalies oriented differently and that depend on the drum rotation.

For each drum, the residual magnetization of the steel sheet that forms the lateral surface of the drum will vanish when the sheet is folded back on itself. One effect of magnetization due to the end covers might actually remain, even after the assembly of the various parts has been completed. This effect becomes evident when a drum lying on the surface is rotated several times as measurements are carried out. The shape of the anomaly is different when the drum is buried, because the measurements are vertical.

\section{The new test site}

A new test site near the INGV Headquarters in Rome was recently established. This test site has features that originated from volcanic formations (the ground surface has a susceptibility of about $6.5 \times 10^{-3} \mathrm{SI}$ ), and it is located in an

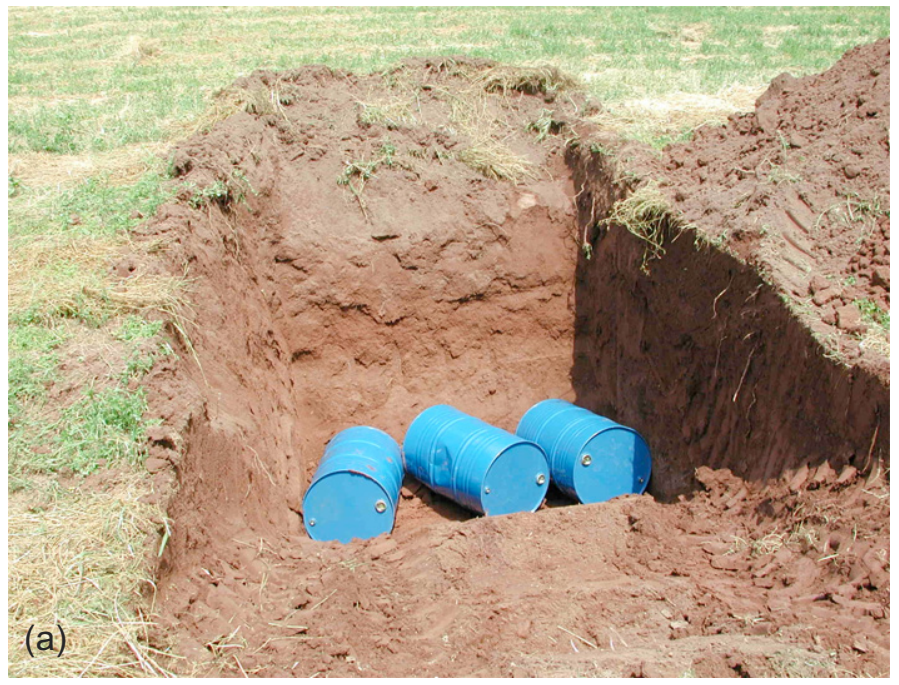

urban area that is today subjected to high electromagnetic interference. Three metal drums were buried at a depth of $2 \mathrm{~m}$. Two more drums, one in a vertical position and the second in a horizontal position, were placed at a safe distance from the other three drums. In addition, a 1-m-length of railway track that weighed about $80 \mathrm{~kg}$ was also buried $1 \mathrm{~m}$ deep.

This site was used for educational purposes, to test innovative geophysical instruments, to refine the techniques for data processing, and to study magnetic anomalies. The anomalies produced by these underground targets were studied by measuring the vertical magnetic gradient on regular grids $(1 \mathrm{~m} \times 1 \mathrm{~m})$. This technique was chosen as it enables the elimination of the electromagnetic interference, which is strong in the temporal variations of the geomagnetic field. The measurements were performed using a Geo-
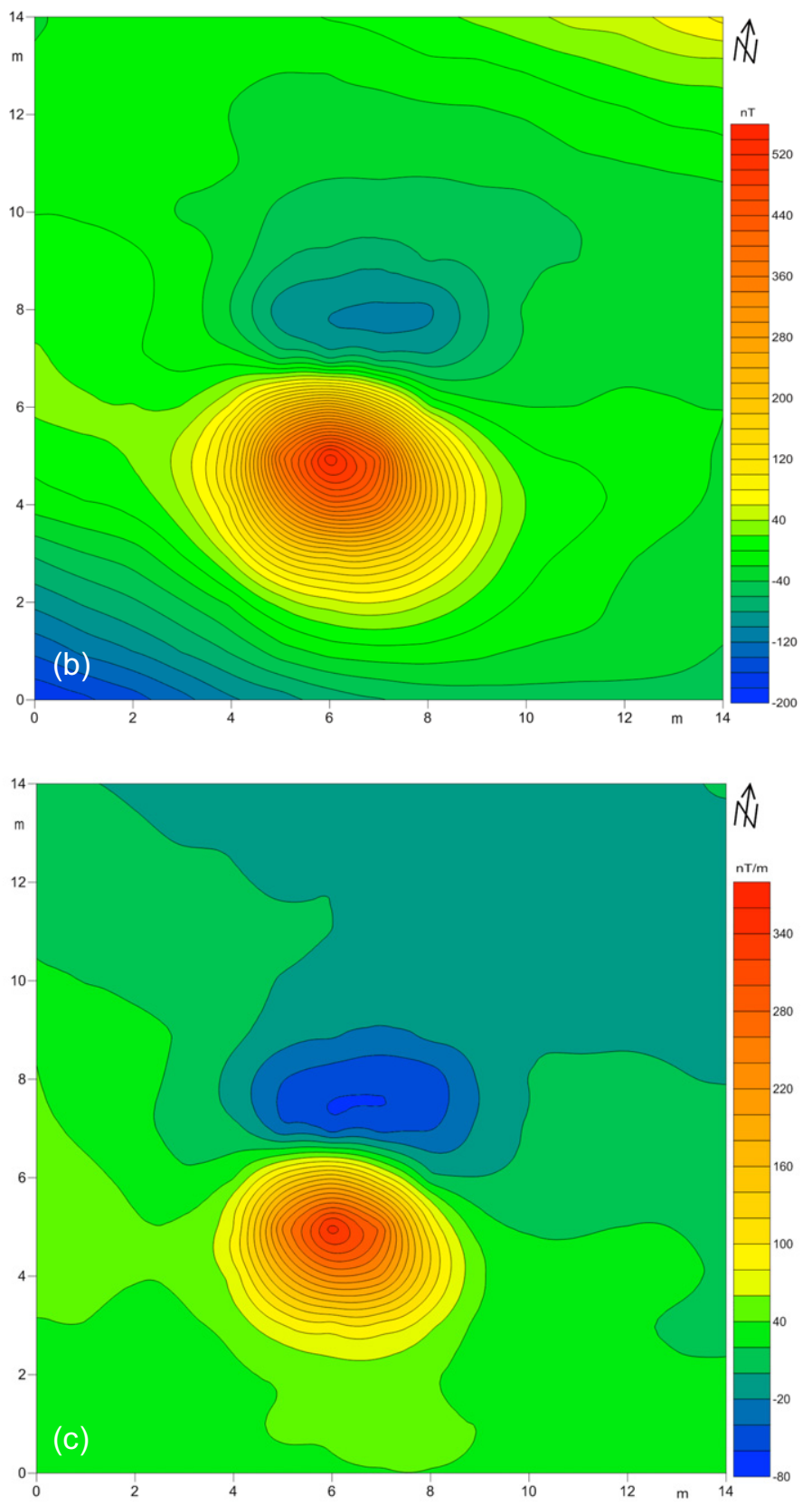
. (a) Photograph of the preparation of the underground target. (b) Anomaly map of the total intensity field. (c) Map of the vertical gradient. 
metrics Cesium optical pumping magnetometer (model G858) with two sensors positioned vertically at $50 \mathrm{~cm}$ and $130 \mathrm{~cm}$ from ground level.

The magnetic anomaly maps were built up from data screened using a low-pass filter and with the application of a suitable polynomial regression to remove electromagnetic noise and the spatial gradients, respectively, caused by the local geology. The data used are from a sensor positioned about 50 $\mathrm{cm}$ above ground level. The vertical gradient maps were instead generated directly, without requiring any special filtering.

Both the magnetic field anomaly and the vertical gradient anomaly produced by the three steel drums were perfectly oriented in the S-N direction, and they reached an intensity of $720 \mathrm{nT}$ and $420 \mathrm{nT} / \mathrm{m}$, respectively. These effects were observed even when the drums were isolated. The anomalies of the vertical magnetic gradient and the total magnetic field intensity for the three drums and for a single drum buried vertically or horizontally are shown in Figures 3, 4 and 5. In particular, a sampling of the vertical gradient distribution, along a N-S oriented profile, is reported in Table 1, across the magnetic anomaly due to the three buried steel drums (Figure 3c).

When a single drum is buried and measurements are performed on the ground surface, the drum appears to consist of three separate pieces (two covers and the cylinder wall): the induced magnetization prevails over the permanent magnetization of the single parts. In the opinion of the authors, the cylindrical part of a drum is predominant over the two lids; indeed, the surface of the cylindrical part is 16400 $\mathrm{cm}^{2}$, which is about 6-fold greater than a single cover area, which is $2789 \mathrm{~cm}^{2}$. Instead, when a single drum is placed on the ground surface and measurements are conducted laterally, as described in Section 4, the drum produces a field anomaly with a different behavior. In this case, the permanent magnetization of each single part produced the most obvious effects, relative to the drum rotation and orientation.

The magnetic anomaly produced by the underground piece of rail track (Figure 6) was E-W oriented, according to the maximum size of the body. This effect was due both to the permanent magnetization of the body and its dimensional anisotropy, as described in Section 2.2.

Figure 7 shows the magnetic anomaly produced by a drum lid placed on the ground surface, and the corresponding anomaly after its $180^{\circ}$ rotation. The direction of the anomaly main axis changes significantly after rotation, meaning that the effects of permanent magnetization are prevalent over induced magnetization.

\section{Digging operations and conclusions}

It is very important to describe the magnetic anomalies produced by buried steel drums, in order to appropriately interpret a magnetic map of a suspected area. The anomalies can be distinguished as fields generated by heterogeneous iron bodies or buried storage drums.
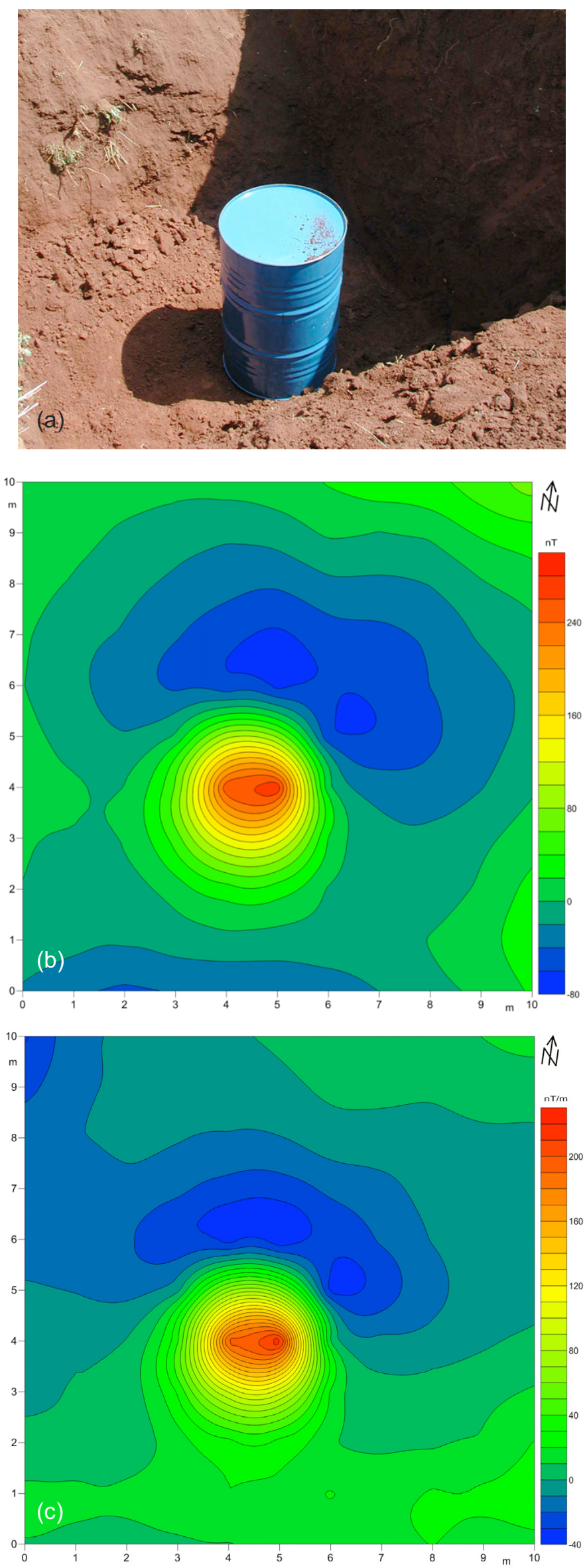

Figure 4. A single drum buried vertically. The magnetic inclination was around $58^{\circ}$. (a) Photograph of the preparation of the underground target. (b) Anomaly map of the total intensity field. (c) Map of vertical gradient. 

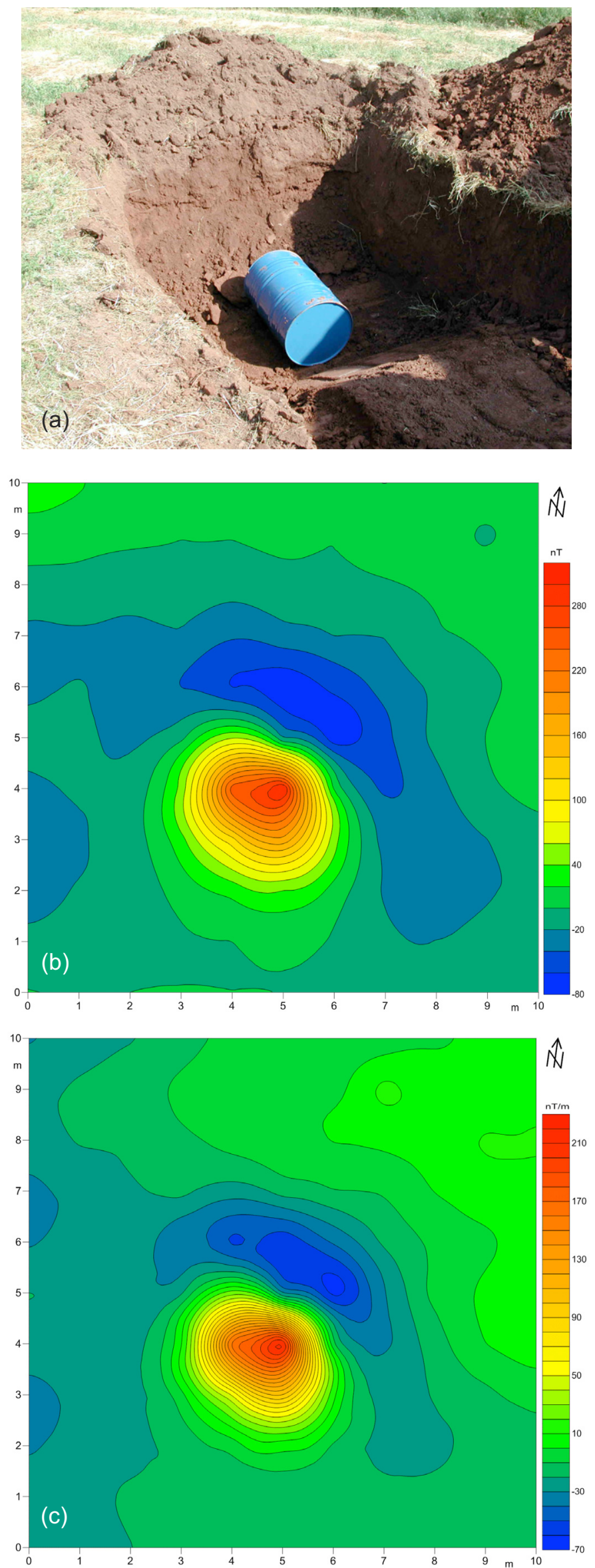

Figure 5. A single drum buried horizontally. The magnetic inclination was around $58^{\circ}$. (a) Photograph of the preparation of the underground target. (b) Anomaly map of the total intensity field. (c) Map of vertical gradient.

\begin{tabular}{|c|c|c|}
\hline $\mathrm{X}$ & $\mathbf{Y}$ & VRT_GRD \\
\hline 6.014 & 0.4 & 40.0 \\
\hline 6.000 & 0.9 & 41.2 \\
\hline 6.000 & 1.4 & 43.8 \\
\hline 6.000 & 1.9 & 50.6 \\
\hline 6.010 & 2.4 & 61.7 \\
\hline 6.023 & 2.9 & 89.5 \\
\hline 6.015 & 3.3 & 145.7 \\
\hline 6.001 & 3.9 & 232.1 \\
\hline 6.009 & 4.3 & 288.8 \\
\hline 6.023 & 4.9 & 344.0 \\
\hline 6.024 & 5.3 & 282.7 \\
\hline 6.024 & 5.9 & 167.1 \\
\hline 6.015 & 6.3 & 71.0 \\
\hline 6.002 & 6.9 & -39.5 \\
\hline 6.009 & 7.3 & -60.5 \\
\hline 6.022 & 7.9 & -52.7 \\
\hline 6.016 & 8.3 & -39.2 \\
\hline 6.002 & 8.9 & -17.1 \\
\hline 6.000 & 9.3 & -10.5 \\
\hline 6.000 & 9.8 & -7.2 \\
\hline 6.008 & 10.3 & -4.1 \\
\hline 6.021 & 10.8 & -0.6 \\
\hline 6.024 & 11.3 & -0.0 \\
\hline 6.024 & 11.8 & -0.2 \\
\hline 6.024 & 12.3 & -3.2 \\
\hline 6.024 & 12.8 & -8.0 \\
\hline 6.024 & 13.2 & -9.6 \\
\hline 6.024 & 13.8 & -10.7 \\
\hline
\end{tabular}

Table. 1. A sampling in $(\mathrm{X}, \mathrm{Y})$ coordinates of the vertical gradient distribution VRT_GRD, along a N-S oriented profile, across the magnetic anomaly due to the three buried steel drums (Figure 3c).

After finding an anomaly that appears related to the presence of steel drums, it is very important to determine the quantity and depth of the iron mass. Much information can be drawn from the morphology, intensity and shape characteristics of anomalies for evaluation of the pertinence of performing an excavation, for estimation of the volume of drums the depth of the deposits, and finally for guiding the excavation, to avoid damaging the drums and dispersing their contents into the environment during the recovery. This assessment must be performed rapidly, since the digging operations often need to be conducted immediately following the geophysical surveys. Therefore, it is necessary to provide immediate guidance for work operators and to define the procedure for excavation, starting from a preliminary magnetic map that is processed on site.

On the basis of our experience from many excavations, 

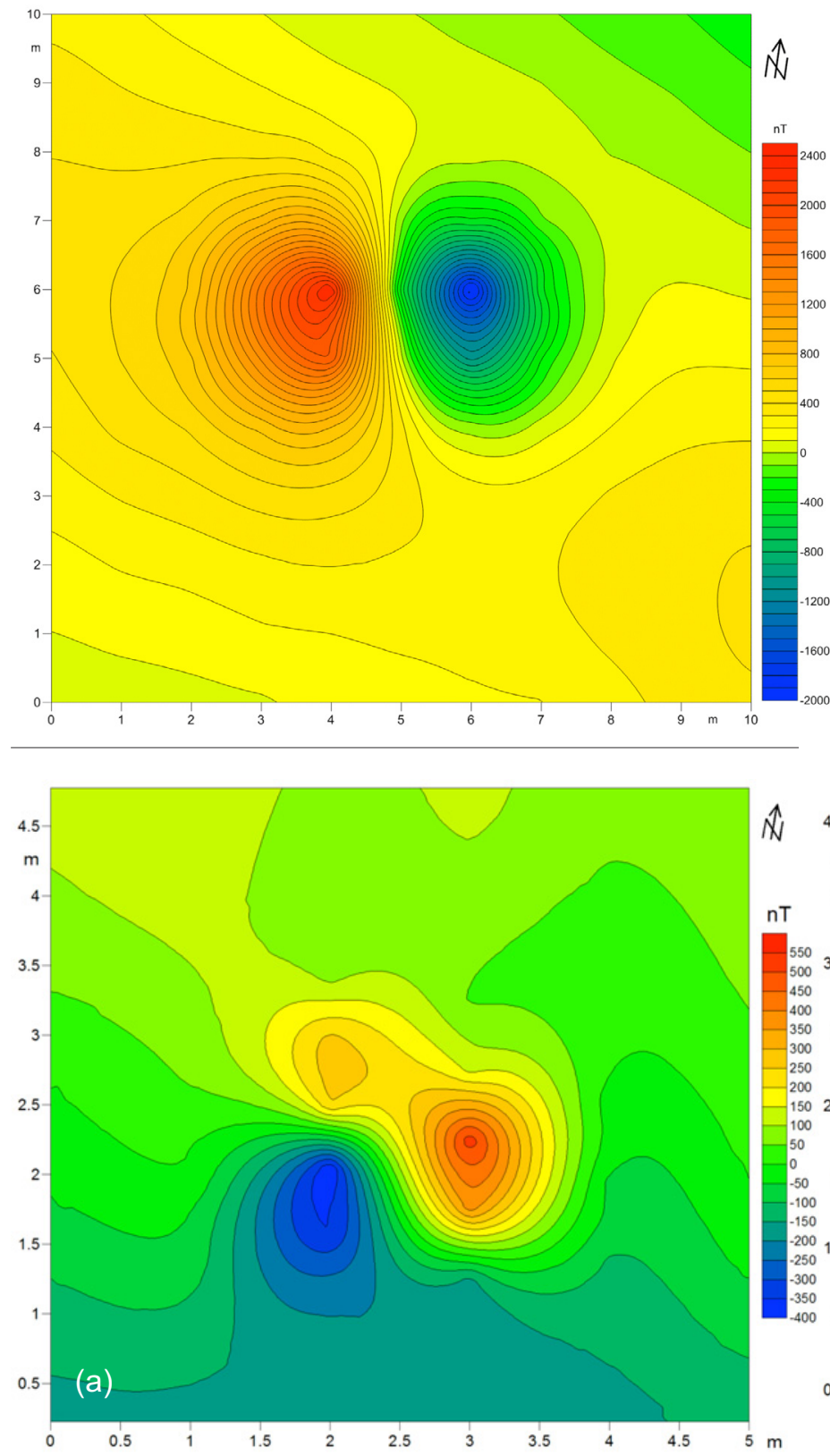

the target depth was assessed as around $90 \%$ of the planimetric distance (in $\mathrm{m}$ ) between the maximum and minimum points on the magnetic anomalies map. This measurement can be related to the distance from the sensor. Therefore, it is essential to have a large number of measurements, sampled on a regular grid, to define the exact morphology of the magnetic anomaly.

Generally, there is no information available on the underground storage of drums, so whether they will be vertical, horizontal, or arranged chaotically. Thus it is even more difficult to provide information about the number of containers when modeling waste disposal sites.

Numerous studies on the magnetic anomalies that can be generated by steel drums have been discussed here. The modeling of magnetic anomalies produced by steel drums with an in-depth analysis of permanent and induced parts has also been presented. Moreover, studies undertaken at the INGV in this field that have made use of field experience and
Figure 6 (left). Underground railway track, as E-W oriented. Anomaly map of the total intensity field.

Figure 7 (below, left to right). A drum lid on the ground surface. (a) Anomaly map of the total field intensity. (b) As (a), after rotation of $180^{\circ}$.

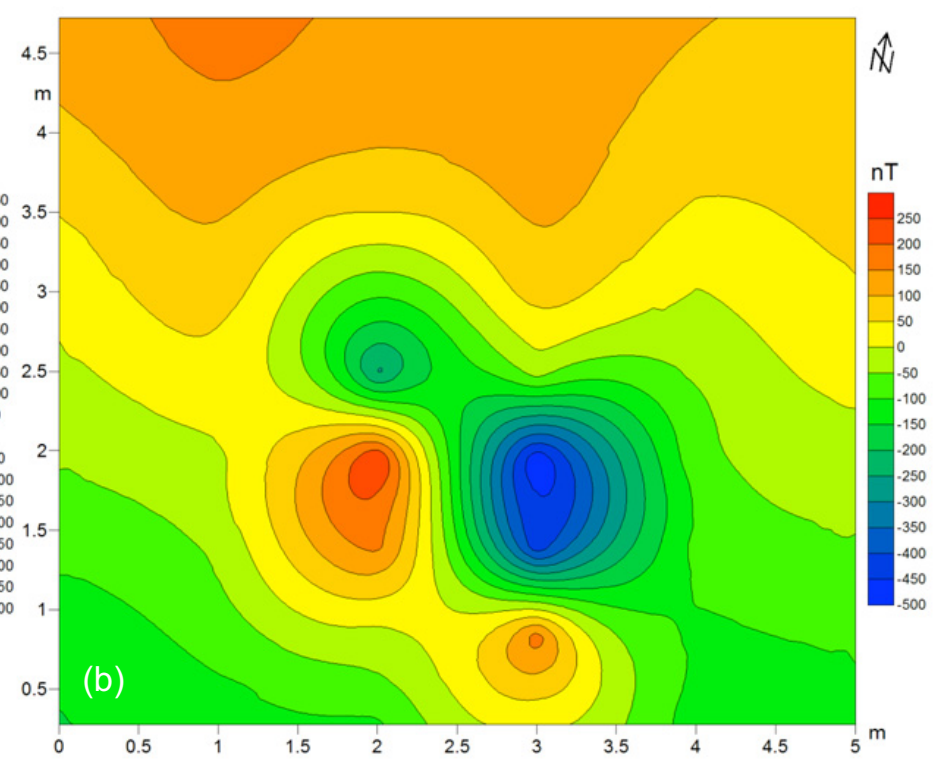

the test sites prepared for this purpose have been shown. The authors hope that this study can serve as a reference for those who are working in this field and are applying magnetometric techniques to detect buried drums in the time consuming and complex activity of environment protection, in which the contribution of geophysics, and in particular of magnetometry, can be very significant.

Acknowledgements. The authors are extremely grateful to Dr. Antonio Meloni for valuable and stimulating suggestions to improve our manuscript and for interesting discussions on magnetic anomalies of steel drums. Moreover, upon request, the authors are willing to provide the entire set of measured data to whom it may concern for further processing, interpretations and/or discussions.

\section{References}

Barrows, L., and J.E. Rocchio (1990). Magnetic surveying for buried metallic objects, Ground Water Monit. Rev., 10, 204-211. 
Bevan, B.W. (1983): Quantitative magnetic analysis of landfill, Geosight Technical Report no. 1.

Breiner, S. (1973). Applications manual for portable magnetometers. Geometrics, 395 Java Drive, Sunnyvale, California, $58 \mathrm{pp}$.

Chianese, D., M. D'Emilio, M. Bavusi, V. Lapenna and M. Macchiato (2006a). Magnetic and ground-probing radar measurements for soil pollution mapping in the industrial area of Val Basento (Basilicata Region, southern Italy): a case study, Environ. Geol., 49, 389-404.

Chianese, D., M. D'Emilio, S. Di Salvia, V. Lapenna, M. Ragosta and E. Rizzo (2006b). Magnetic mapping, ground penetrating radar surveys and magnetic susceptibility measurements for the study of the archaeological site of Serra di Vaglio (southern Italy), J. Arch. Sci., 31, 633-643.

Cochran, J.R., and K.E. Dalton (1995). Using high-density magnetic and electromagnetic data for waste characterization, a case study, In: Proceedings of SAGEEP, edited by R.S. Bell, EEGS, Orlando, Florida, p. 117.

Emerson, D.W., J.E. Reid, D.A. Clark, M.S.C. Hallett and P.B. Manning (1992). The geophysical responses of buried drums - field tests in weathered Hawkesbury Sandstone, Sydney Basin, NSW, Explor. Geophys., 23, 589-617.

Eskola, L., H. Soininen and M. Oksama (1989). Modelling of resistivity and IP anomalies of a thin conductor with an integral equation, Geoexploration, 26, 95-104.

Eskola, L. (1992). Geophysical Interpretation Using Integral Equations, edited by Chapman \& Hall, London, 191 pp.

Eskola, L., T. Jokinen, H. Soininen and T. Tervo (1993). Some remarks on static field thin sheet models, J. Appl. Geophys., 30, 229-234.

Eskola, L., R. Puraner and H. Soininen (1999). Measurement of magnetic properties of steel sheets, Geophys. Prospect., 47, 593-602.

Foley, J.E. (1994). STOLMTM Magnetic survey at Sandia National Laboratory Tecnical area 2, In: Proceedings of SAGEEP, edited by R.S. Bell and C.M. Lepper, March 2731, 1994, Boston, Massachusetts, 895-907.

Frischknecht, E.C., and P.V. Raab (1985). Location of abandoned wells with geophysical methods: National Technical Information Service (NTIS) Report PB 85-122638, Springfield, Virginia, 48 pp.

Furness, P. (1999). A versatile integral equation technique for magnetic modelling, J. Appl. Geophys., 41, 345-357.

Furness, P. (2001). A note on magnetic modelling with remanence, J. Appl. Geophys., 48, 257-261.

Furness, P. (2002). The magnetic field of steel drums, J. Appl. Geophys., 51, 63-74.

Furness, P. (2007). Modelling magnetic fields due to steel drum accumulation, Geophys. Prospect., 55, 737-748.

Gibson, P.J., P. Lyle and D.M. Gorge (1996). Environmental applications of magnetometry profiling, Environ. Geol., 27, 178-183.
Gilkeson, R.H., P.C. Heigold and D.E. Laymon (1986). Practical application of theoretical models to magnetometer surveys on hazardous waste disposal sites - A case history, Ground Water Monit. Rev., 6, 54-61.

Gilkeson, R.H., S.R. Gorin and D.E. Laymon (1992). Application of magnetic and electromagnetic methods to metal detection, In: Proceedings of SAGEEP, edited by R.S. Bell, EEGS, April 26-29, 1992, Oakbrook, Illinois, 309-328.

Godio, A., M.C. Zanetti and L. Giordanetto (1999). Geophysical site investigation for "landfill mining", In: Processings Sardinia 99, Seventh International Waste Management and Landfill Symposium, October 4-8, 1999, S. Margherita di Pula, Cagliari, Italy, 4, 587-594.

Godio, A. (2000). Magnetic data interpretation in an industrial waste landfill, Annali di Geofisica, 43 (2), 297-307.

Goldstein, N.E., and S.H. Ward (1966). The separation of remanent from induced magnetism in situ, Geophysics, 31, 779-796.

Grant, F.S., and G.F. West (1965). Interpretation Theory in Applied Geophysics, edited by McGraw-Hill, New York, 306-381.

Gubbins, D., and E. Herrero-Bervera (2007). Encyclopedia of Geomagnetism and Paleomagnetism, Series: Encyclopedia of Earth Sciences Series, edited by Springer, Hardcover, XXVI, $1054 \mathrm{pp}$.

Huang, H., and D.A. Keiswetter (1997). Comparison of magnetic and electromagnetic data for underground structures, J. Environ. Eng. Geophys., 2, 115-126.

Jachens, R.C., M.W. Webring and E.C. Frischknecht (1986). Abandoned-well study in the Santa Clara Valley, California, U.S. Geological Survey, Open-File Report 86-350, 13 pp.

Jackson, M. (1991). Anisotropy of magnetic remanence: a brief review of mineralogical sources, physical origins, and geological applications, and comparison with susceptibility anisotropy, Pure Appl. Geophys., 36, 1-28.

Lanza, R., and A. Meloni (2006). The Earth's Magnetism - An Introduction for Geologists, edited by Springer-Verlag, 1st edition, Berlin, Germany, 290 pp.

Marchetti, M., M. Chiappini and A. Meloni (1996). Anomalie magnetiche generate da fusti metallici: gli effetti prodotti da un singolo fusto, In: Atti XV Convegno Nazionale CNR-GNGTS, Roma, 5-12 (in Italian).

Marchetti, M. (1997). Applicazioni della magnetometria alle problematiche ambientali, in particolare alle discariche, Geologia dell'ambiente, periodico della Società Italiana di Geologia Ambientale, 4, 20-23 (in Italian).

Marchetti, M., and A. Meloni (1997). Ricerca di corpi metallici sepolti con tecniche magnetometriche. Una breve rassegna, In: Atti XVI Convegno Nazionale CNRGNGTS, Roma (in Italian).

Marchetti, M., M. Chiappini and A. Meloni (1998). A test site for magnetic detection of buried steel drums, Annali di Geofisica, 41 (3), 491-498. 
Marchetti, M. (2000). Anomalie magnetiche in aree di discarica: una breve rassegna, In: Atti XIX Convegno Nazionale CNR-GNGTS, Roma (in Italian).

Marchetti, M., L. Cafarella, D. Di Mauro and A. Zirizzotti (2002). Ground magnetometric survey and integrated geophysical methods for solid buried waste detection: a case study, Annals of Geophysics, 45 (3/4), 563-573.

Marchetti, M., and A. Settimi (2011). Integrated geophysical measurements on a test site for detection of buried steel drums, Annals of Geophysics, 54 (1), 105-114.

Nabigian, M.N., G.L. Oppliger, R.N. Edwards, B.B.H. Lo and S.J. Cheesman (1984). Cross-hole magnetometric resistivity (MMR), Geophysics, 49, 1313-1326.

Nettleton, L.L. (1976). Gravity and Magnetics in Oil Prospecting, McGraw-Hill, New York, 305-426.

Parasnis, D.S. (1986). Mining Geophysics, Elsevier Scientific Publishing Co., Amsterdam, 3-60.

Parkinson, W.D. (1983). Introduction to Geomagnetism, edited by Elsevier Science Pub. Co., Inc., New York, 434 pp.

Phillips, H.B. (1934). Effect of surface discontinuity on the distribution of potential, J. Math. Phys., 13, 261-267.

Pierce, D., and J. De Reamer (1993). Geophysical investigation for buried drums: a case study, In: Proceedings of SAGEEP, edited by R.S. Bell and C.M. Lepper, EEGS, San Diego, California, 229-244.

Ravat, D. (1996). Magnetic properties of unrusted steel drums from laboratory and field magnetic measurements, Geophysics, 61, 1325-1335.

Ravat, D., A. Pignatelli, I. Nicolosi and M. Chiappini (2007). A study of spectral methods of estimating the depth to the bottom of magnetic sources from near-surface magnetic anomaly data, Geophys. J. Int., 169, 421-434.

Rikitake, T., and Y. Honkura (1985). Solid Earth Geomagnetism, edited by Terra Scientific, $384 \mathrm{pp}$.

Roberts, R.L., W.J. Hinze and D.I. Leap (1990). Data enhancement procedures on magnetic data from landfill investigations, edited by S. H. Ward, Soc. Expl. Geophys., Geotech. Environ. Geophys., 261-266.

Robinson, E.S., and C. Coruh (1988). Basic Exploration Geophysics, J. Wiley \& Sons, New York, 333-444.

Salem, A., D. Ravat, T.J. Gamey and K. Ushijima (2002). Analytic signal approach and its applicability in environmental magnetic investigations, J. Appl. Geophys., 49, 231-244.

Schlinger, C.M. (1990). Magnetometer and gradiometer surveys for detection of underground storage tanks, Bull Assoc. Eng. Geol., 27, 37-50.

Sheinker, A., L. Frumkis, B. Ginzburg, N. Salomonski and B.Z. Kaplan (2009). Magnetic anomaly detection using a three-axis magnetometer, IEEE T. Magn., 45, 160-167.

Singh, S.K., and F.J. Sabina (1978). Magnetic anomaly due to a vertical right circular cylinder with arbitrary polarization, Geophysics, 43, 173-178.
S.E.G. (Soc. of Exp. Geophysicists eds.) (1967). Mining Geophysics, vol. II, Soc. of Exp. Geophysicists, Tulsa, Oklahoma, 423-620.

Stratton, J.A. (1941). Electromagnetic Theory, edited by McGraw-Hill, New York, 615 pp.

Telford, W.M., L.P. Geldart, R.E. Shrift and D.A. Keys (1976). Applied Geophysics, edited by Cambridge University Press, Cambridge, 105-217.

Traynin, P., and R.O. Hansen (1993). Magnetic modeling for highly permeable bodies with remanent magnetization, SEG Expanded Abstract, 12, 410-413; doi:10.1190/1.1894 192.

Tyagi, S., A.E. Lord Jr. and R.M. Koerner (1983). Use of a proton precession magnetometer to detect buried drums in sandy soil, J. Hazard. Mater., 8, 11-23.

Vogelsang, D. (1994). Environmental Geophysics. A Practical Guide, Springer-Verlag Eds., 1916 pp.

*Corresponding author: Marco Marchetti, Istituto Nazionale di Geofisica e Vulcanologia, Sezione di Geomagnetismo, Aeronomia e Geofisica Ambientale, Rome, Italy; email: marco.marchetti@ingv.it.

(C) 2013 by the Istituto Nazionale di Geofisica e Vulcanologia. All rights reserved. 